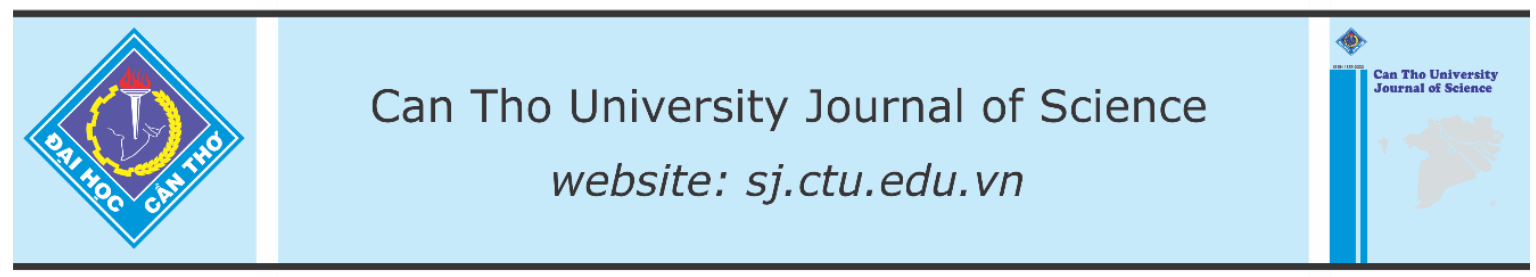

DOI: $10.22144 /$ ctu.jen.2021.029

\title{
Synchronization in complete networks of reaction-diffusion equations of FitzHugh- Nagumo type with nonlinear coupling
}

\author{
Phan Van Long Em* \\ An Giang University, Viet Nam National University, Ho Chi Minh City \\ *Correspondence: Phan Văn Long Em (email: pvlem@agu.edu.vn)
}

\section{Article info.}

Received 22 Sep 2020

Revised 29 Oct 2020

Accepted 19 Jul 2021

\section{Keywords}

Complete network, coupling strength, FitzHugh-Nagumo model, synchronization

\section{ABSTRACT}

The synchronization in complete network consisting of $n$ nodes is studied in this paper. Each node is connected to all other ones by nonlinear coupling and is represented by a reaction-diffusion system of FitzHughNagumo type which can be obtained by simplifying the famous HodgkinHuxley model. From this complete network, the sufficient condition on the coupling strength to achieve the synchronization is found. The result shows that the networks with bigger in-degrees of nodes synchronize more easily. The paper also presents the numerical simulations for theoretical result and shows a compromise between the theoretical and numerical results.

\section{INTRODUCTION}

Synchronization is a ubiquitous feature in many natural systems and nonlinear science. The word "synchronization" is of Greek origin, with syn as "common" and chronos as "time", which means having the same behavior at the same time. Therefore, the synchronization of two dynamical systems usually means that one system copies the movement of the other. When the behaviors of many systems are synchronized, these systems are called synchronous. Aziz-Alaoui (2006) and Corson (2009) suggested that a phenomenon of synchronization may appear in a network of many weakly coupled oscillators. A broad variety of applications have emerged to increase the power of lasers, synchronize the output of electric circuits, control oscillations in chemical reactions or encode electronic messages for secure communications (AzizAlaoui, 2006; Pikovsky et al., 2001).

In recent years, the synchronization has been extensively studied in many fields, many natural phenomena also reflect the synchronization such as the movement of birds forming the cloud, the move- ment of fishes in the lake, the movement of the parade, the reception and transmission of a group of cells, etc. (Aziz-Alaoui, 2006; Ermentrout \& Terman, 2009; Hodgkin \& Huxley, 1952; Izhikevich, 2005; Murray, 2002). Therefore, the study of the synchronization in the network of cells is very necessary. In order to make the study easier, the complete network of $n$ neurons interconnected together with non-linear coupling is investigated and the sufficient condition on the coupling strength is sought to achieve the synchronization. Each neuron is represented by a dynamical system named FitzHugh-Nagumo model. It was introduced as a dimensional reduction of the well-known Hodgkin-Huxley model (Ermentrout, 2009; Hodgkin, 1952; Izhikevich, 2007; Keener, 2009; Murray, 2002; Nagumo, 1962). It is more analytically tractable and maintains some biophysical meaning. The model is constituted a common form of two equations in the two variables $u$ and $v$. The first variable is the fast one called excitatory which represents the transmembrane voltage. The second is the slow recovery variable which describes the time dependence of several physical quantities, 
such as electrical conductivity of ion currents across the membrane. The FitzHugh-Nagumo equations (FHN) are given by

$$
\left\{\begin{array}{l}
\varepsilon \frac{d u}{d t}=f(u)-v \\
\frac{d v}{d t}=a u-b v+c
\end{array}\right.
$$

where, $a, b$ and $c$ are constants ( $a$ and $b$ are strictly positive), $0<\varepsilon \ll 1$ and $f(u)=-u^{3}+3 u$.

However, this equation is not strong enough to describe the propagation of action potential. To solve this problem, the cable equation is investigated. It is a mathematical equation derived from a circuit model of the membrane and its intracellular and extracellular space to provide a quantitative description of current flow and voltage change both within and between neurons, allowing a quantitative and qualitative understanding of how neurons function. So, in this study, the following partial differential equations (PDE) is considered:

$$
\left\{\begin{array}{l}
\varepsilon \frac{d u}{d t}=\varepsilon u_{t}=f(u)-v+d_{u} \Delta u \\
\frac{d v}{d t}=v_{t}=a u-b v+c
\end{array}\right.
$$

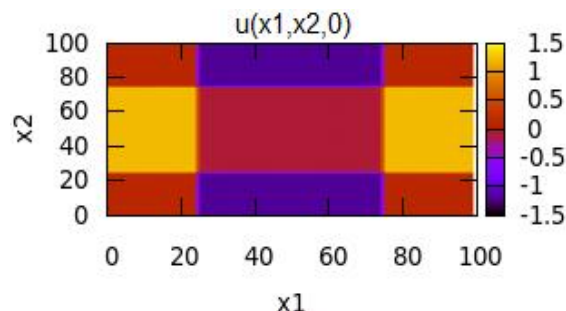

(a) where, $u=u(x, t), v=v(x, t),(x, t) \in \Omega \times \mathbb{R}^{+}, d_{u}$ is a positive constant, $\Delta u$ is the Laplace operator of $u, \Omega \subset \mathbb{R}^{N}, N \in \mathbb{Z}^{+}$is a regular bounded open set and with Neumann zero flux boundary conditions. This system allows the emergence of a variety of patterns and relevant phenomena in physiology (Ambrosio \& Aziz-Alaoui, 2012; Ambrosio \& Aziz-Alaoui, 2013). It is a system of two nonlinear partial differential equations of incomplete parabolic type which describes the action potential and the recovery variable in the whole set of neurons. It is noted that the first equation is similar to the socalled cable equation, which describes the distribution of the potential along the axon of a single neuron (Ermentrout, 2009; Izhikevich, 2005). For example, in figure 1 , there are two solutions of system (1) corresponding to different values of $t$ on space $\Omega=[0 ; 100] \times[0 ; 100]$. Figure 1(a) represents, for $t=0$, the isovalues $u\left(x_{1}, x_{2}, 0\right)$ of one solution of system (1). Figure 1(b) represents, for $t=190$, the isovalues $u\left(x_{1}, x_{2}, 190\right)$ of one solution of system (1). Such a solution is called spiral one obtained by a particular choice of initial conditions.

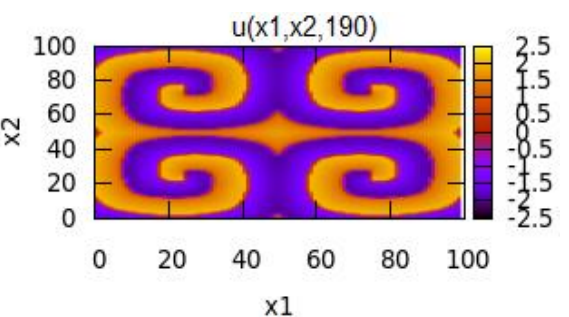

(b)

Figure 1. Figure (a) represents, for $\mathbf{t}=\mathbf{0}$, the isovalues $u\left(x_{1}, x_{2}, 0\right)$ of one solution of system (1). Figure (b) represents, for $\mathbf{t}=190$, the isovalues $u\left(x_{1}, x_{2}, 190\right)$ of one solution of system (1). Such a solution is called spiral one obtained by a particular choice of initial conditions

After having the model of a neuron, we consider a network of $n$ coupled systems (1) based on FHN type as follows:

$$
\begin{aligned}
& \left\{\begin{array}{l}
\varepsilon u_{i t}=f\left(u_{i}\right)-v_{i}+d_{u_{i}} \Delta u_{u_{i}}-h\left(u_{i}, v_{i}\right) \\
v_{i t}=a u_{i}-b v_{i}+c
\end{array}\right. \\
& i, j=1, \ldots, n, i \neq j,
\end{aligned}
$$

where, $\left(u_{i}, v_{i}\right), i=1,2, \ldots, n$ is defined by (1).

The function $h$ is the coupling function that determines the type of connection between neurons $i$ and $j$. Connections between neurons are essentially of two types: chemical which is much more abundant and electrical. If the connections are made by chemical synapse, the coupling is non-linear and given by the function: 


$$
\begin{aligned}
& h\left(u_{i}, v_{i}\right)=\left(u_{i}-V_{s y n}\right) g_{s y n} \sum_{j=1}^{n} c_{i j} \Gamma\left(u_{j}\right), \\
& i=1,2, \ldots, n .
\end{aligned}
$$

The parameter $g_{\text {syn }}$ represents the coupling strength. The coefficients $c_{i j}$ are the elements of the connectivity matrix $C_{n}=\left(c_{i j}\right)_{n \times n}$, defined by

$\left\{\begin{array}{l}c_{i j}=1 \text { if } \mathrm{i} \text { and } \mathrm{j} \text { are coupled } \\ c_{i j}=0 \text { if } \mathrm{i} \text { and } \mathrm{j} \text { are not coupled }\end{array}\right.$

$i, j=1,2, \ldots, n, i \neq j$.

The function $\Gamma$ is a non-linear threshold function

$$
\Gamma\left(u_{j}\right)=\frac{1}{1+\exp \left(-\lambda\left(u_{j}-\theta_{s y n}\right)\right)} .
$$

The parameters have the following physiological meanings:

$V_{s y n}$ is the reversal potential and must be larger than $u_{i}(x, t)$, for all $i, x \in \Omega, t \geq 0$ since synapses are supposed excitatory.

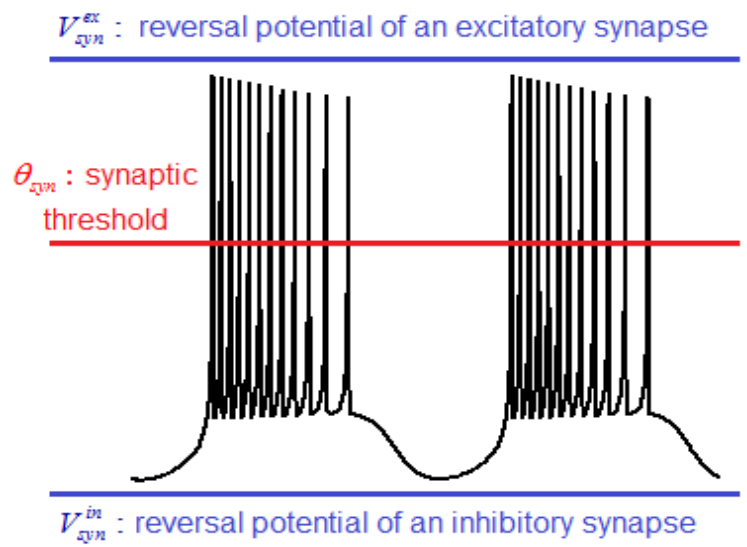

(a) $\theta_{s y n}$ is the threshold reached by every action potential for a neuron.

$\lambda$ is a positive number (Belykh et al., 2005; Corson, 2009). The bigger $\lambda$ is and the better we approach the Heaviside function.

Corson (2009) is interested in the generation of the burst oscillations from a network of equations on Hindmarsh-Rose type. The values of these parameters are determined numerically. For example, $\theta_{s y n}$ is chosen as a central value in the middle of bursts over a given period. Figure 2 represents the values of parameters $\theta_{s y n}$ and $V_{s y n}$ on time series and a 2dimensional projection of phase portrait. The parameter $\theta_{s y n}$ is the synaptical threshold, on the scale time series (a) and on the phase portrait projected on the plane (b). So the value of the membrane potential beyond which the synapse is activable. It is usually chosen "in the middle" of spikes. The parameters $V_{s y n}^{e x}$ and $V_{s y n}^{\text {in }}$ are the "reversal potential". The value of $V_{s y n}^{e x}\left(\operatorname{resp} . V_{s y n}^{i n}\right)$ is bigger (resp. smaller) than the maximal value (resp. minimal) of action potential and is used in the modeling of excitatory synapses (resp. inhibitory).

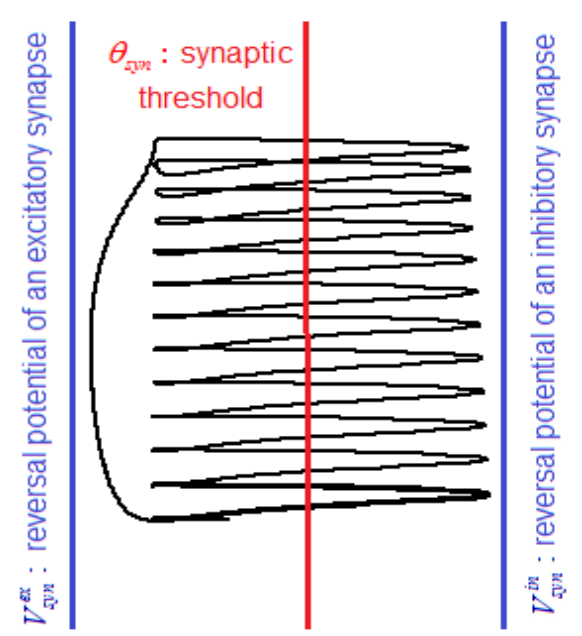

(b)

Figure 2. The parameter $\theta_{s y n}$ is the synaptical threshold, on the scale time series (a) and on the phase portrait projected on the plane (b)

In recent years, there are a lot of studies concerning to the synchronization; however, they are just studied for the linear coupling, while the connections between neurons made by chemical synapse is ma- jor in neural network. Therefore, it is really useful to research about this problem. In other words, the rapid chemical excitatory synapses are interested, so the parameters are fixed as follows throughout 
this paper, according to the articles (Belykh et al., 2005; Corson, 2009).

$$
\lambda=10, V_{\text {syn }}=2, \theta_{\text {syn }}=-0,25 .
$$

A neural network describes a population of physically interconnected nerve cells. Communication between cells is mainly due to electrochemical processes. This article focuses on analyzing the behavior of a set of neurons connected with a given topology by chemical signals. Thus, the complex system based on a network of interactions between neurons is considered in which each network node is modeled by a PDE of FHN type. The sufficient conditions to obtain the synchronization in network are found, and the minimal value of coupling strength to get the synchronization is investigated by numerical experiments, that gives an insight into the influence of neurons on minimal coupling strength. The numerical simulations show that when the number of nodes in graph grows, the network becomes easier to synchronize.

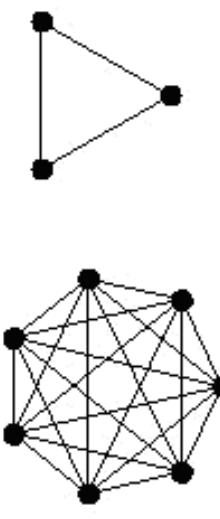

\section{SYNCHRONIZATION OF A COMPLETE NETWORK OF N SYSTEMS OF REACTION-DIFFUSION ON FITZHUGH- NAGUMO TYPE WITH NONLINEAR COUPLING}

In this article, the synchronization is investigated in complete network which means that each node connects to all other nodes of the network (Ambrosio \& Aziz-Alaoui, 2012, 2013). For example, Figure 3 shows the complete graphs from 3 to 10 nodes. In this study, each node represents a neuron modeled by a system of reaction-diffusion equations on FHN type and each edge represents a synaptic connection modeled by a coupling function.

Definition 1. Let $S_{i}=\left(u_{i}, v_{i}\right), i=1,2, \ldots, n$ and $S=\left(S_{1}, S_{2}, \ldots, S_{n}\right)$ be a network. We say that $S$ is synchronous if

$$
\lim _{t \rightarrow+\infty} \sum_{i=1}^{n-1}\left(\left\|u_{i}-u_{i+1}\right\|_{L^{2}(\Omega)}+\left\|v_{i}-v_{i+1}\right\|_{L^{2}(\Omega)}\right)=0 .
$$
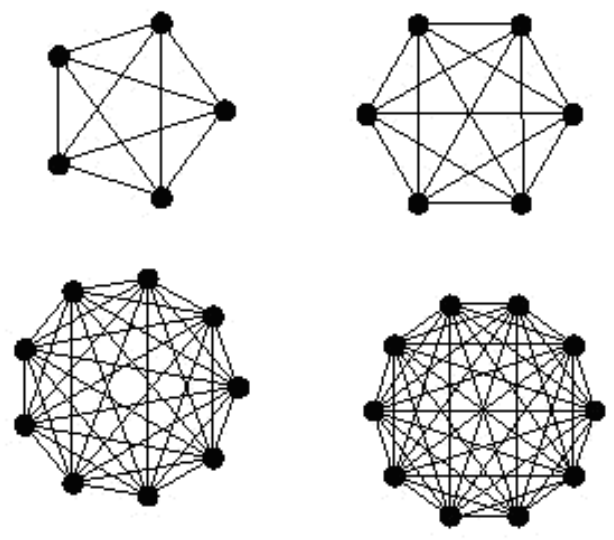

Figure 3. Complete graphs from 3 to 10 nodes

A system of $n$ "neurons" (1) bi-directionally coupled by the chemical synapses, based on FHN, is given as follows:

$$
\left\{\begin{array}{l}
\varepsilon u_{i t}=f\left(u_{i}\right)-v_{i}+d_{u_{i}} \Delta u_{i}-\sum_{k=1, k \neq i}^{n} \frac{g_{n}\left(u_{i}-V_{\text {syn }}\right)}{1+\exp \left(-\lambda\left(u_{k}-\theta_{\text {syn }}\right)\right)} \quad i=1,2, \ldots, n, \\
v_{i t}=a u_{i}-b v_{i}+c
\end{array}\right.
$$

where, $g_{n}$ is the coupling strength between $u_{i}$ and $u_{j}$.

Theorem 1. Suppose that

$$
N=\inf \left\{u_{i}(x, t), i=1,2, \ldots, n, x \in \Omega, t \geq 0\right\}, d_{u_{i}}=d_{u_{j}}, i, j=1,2, \ldots, n
$$


And $g_{n}>\frac{M\left[1+\exp \left(-\lambda\left(N-\theta_{\text {syn }}\right)\right)\right]}{n-1}$,

where $M=\sup _{u \in B, x \in \mathbb{R}} \sum_{k=1}^{3} \frac{f^{(k)}(u)}{k !} x^{k-1}$, B is a compact interval including $\mathrm{u}$ and $f^{(k)}(u)$ is the kth derivative of $\mathrm{f}$ with respect to $u$. Then the network (4) synchronizes in the sense of Definition 1.
Proof.

Let $\Phi(t)=\frac{1}{2}\left[\sum_{i=2}^{n}\left(a \varepsilon \int_{\Omega}\left(u_{i}-u_{1}\right)^{2} d x+\int_{\Omega}\left(v_{i}-v_{1}\right)^{2} d x\right)\right]$.

By deriving the function $\Phi(t)$, there is the following

$$
\begin{aligned}
& \frac{d \Phi(t)}{d t}=\sum_{i=2}^{n} \int_{\Omega}\left[a \varepsilon\left(u_{i}-u_{1}\right)\left(u_{i t}-u_{1 t}\right)+\left(v_{i}-v_{1}\right)\left(v_{i t}-v_{1 t}\right)\right] d x \\
& =\sum_{i=2}^{n} \int\left[a ( u _ { i } - u _ { 1 } ) \left(f\left(u_{i}\right)-v_{i}+d_{u_{i}} \Delta u_{i}-\sum_{k=1, k \neq i}^{n} \frac{g_{n}\left(u_{i}-V_{\text {syn }}\right)}{1+\exp \left(-\lambda\left(u_{k}-\theta_{\text {syn }}\right)\right)}-f\left(u_{1}\right)+v_{1}-d_{u_{1}} \Delta u_{1}\right.\right. \\
& \left.\left.+\sum_{l=2}^{n} \frac{g_{n}\left(u_{1}-V_{\text {syn }}\right)}{1+\exp \left(-\lambda\left(u_{l}-\theta_{\text {syn }}\right)\right)}\right)+\left(v_{i}-v_{1}\right)\left(a\left(u_{i}-u_{1}\right)-b\left(v_{i}-v_{1}\right)\right)\right] d x \\
& =\sum_{i=2}^{n} \int_{\Omega}\left[a ( u _ { i } - u _ { 1 } ) \left(f\left(u_{i}\right)+d_{u_{i}} \Delta u_{i}-f\left(u_{1}\right)-d_{u_{1}} \Delta u_{1}-\sum_{k=1, k \neq i}^{n} \frac{g_{n}\left(u_{i}-V_{\text {syn }}\right)}{1+\exp \left(-\lambda\left(u_{k}-\theta_{\text {syn }}\right)\right)}\right.\right. \\
& \left.\left.+\sum_{l=2}^{n} \frac{g_{n}\left(u_{1}-V_{\text {syn }}\right)}{1+\exp \left(-\lambda\left(u_{l}-\theta_{\text {syn }}\right)\right)}\right)-b\left(v_{i}-v_{1}\right)^{2}\right] d x \\
& \leq \sum_{i=2}^{n} \int_{\Omega}\left[a ( u _ { i } - u _ { 1 } ) \left(f\left(u_{i}\right)-f\left(u_{1}\right)-\sum_{k=1, k \neq i}^{n} \frac{g_{n}\left(u_{i}-V_{s y n}\right)}{1+\exp \left(-\lambda\left(u_{k}-\theta_{s y n}\right)\right)}\right.\right. \\
& \left.\left.+\sum_{l=2}^{n} \frac{g_{n}\left(u_{1}-V_{\text {syn }}\right)}{1+\exp \left(-\lambda\left(u_{l}-\theta_{\text {syn }}\right)\right)}\right)-b\left(v_{i}-v_{1}\right)^{2}\right] d x \\
& \leq \sum_{i=2}^{n} \int_{\Omega}\left[a ( u _ { i } - u _ { 1 } ) \left(f\left(u_{i}\right)-f\left(u_{1}\right)-\sum_{k=1, k \neq i}^{n} \frac{g_{n}\left(u_{i}-u_{1}\right)}{1+\exp \left(-\lambda\left(u_{k}-\theta_{s y n}\right)\right)}\right.\right. \\
& \left.\left.+g_{n}\left(u_{1}-V_{\text {syn }}\right)\left(\sum_{l=2}^{n} \frac{1}{1+\exp \left(-\lambda\left(u_{l}-\theta_{\text {syn }}\right)\right)}-\sum_{k=1, k \neq i}^{n} \frac{1}{1+\exp \left(-\lambda\left(u_{k}-\theta_{\text {syn }}\right)\right)}\right)\right)-b\left(v_{i}-v_{1}\right)^{2}\right] d x \\
& \leq \sum_{i=2}^{n} \int_{\Omega}\left[a ( u _ { i } - u _ { 1 } ) \left(f\left(u_{i}\right)-f\left(u_{1}\right)-\sum_{k=1, k \neq i}^{n} \frac{g_{n}\left(u_{i}-u_{1}\right)}{1+\exp \left(-\lambda\left(u_{k}-\theta_{s y n}\right)\right)}\right.\right. \\
& \left.\left.+g_{n}\left(u_{1}-V_{\text {syn }}\right)\left(\frac{1}{1+\exp \left(-\lambda\left(u_{i}-\theta_{\text {syn }}\right)\right)}-\frac{1}{1+\exp \left(-\lambda\left(u_{1}-\theta_{\text {syn }}\right)\right)}\right)\right)-b\left(v_{i}-v_{1}\right)^{2}\right] d x .
\end{aligned}
$$

Since we are interested in the rapid chemical excitatory synapses, so

$$
\begin{aligned}
& u_{1}<V_{s y n}, \forall x \in \Omega, t \geq 0 \Rightarrow \\
& u_{1}-V_{\text {syn }}<0, \forall x \in \Omega, t \geq 0 .
\end{aligned}
$$


Note that:

- If $u_{i}>u_{1}$, then $u_{i}-u_{1}>0 \Rightarrow g_{n}\left(u_{i}-u_{1}\right)\left(u_{1}-V_{\text {syn }}\right)<0$, and

$$
\frac{1}{1+\exp \left(-\lambda\left(u_{i}-\theta_{\text {syn }}\right)\right)}>\frac{1}{1+\exp \left(-\lambda\left(u_{1}-\theta_{\text {syn }}\right)\right)} \text {. }
$$

Thus

$$
g_{n}\left(u_{i}-u_{1}\right)\left(u_{1}-V_{\text {syn }}\right)\left(\frac{1}{1+\exp \left(-\lambda\left(u_{i}-\theta_{\text {syn }}\right)\right)}-\frac{1}{1+\exp \left(-\lambda\left(u_{1}-\theta_{\text {syn }}\right)\right)}\right)<0 .
$$

- If $u_{i}<u_{1}$, then

$u_{i}-u_{1}<0 \Rightarrow g_{n}\left(u_{i}-u_{1}\right)\left(u_{1}-V_{s y n}\right)>0$,

and

$$
\frac{1}{1+\exp \left(-\lambda\left(u_{i}-\theta_{\text {syn }}\right)\right)}<\frac{1}{1+\exp \left(-\lambda\left(u_{1}-\theta_{\text {syn }}\right)\right)} .
$$

Thus,

$$
g_{n}\left(u_{i}-u_{1}\right)\left(u_{1}-V_{\text {syn }}\right)\left(\frac{1}{1+\exp \left(-\lambda\left(u_{i}-\theta_{\text {syn }}\right)\right)}-\frac{1}{1+\exp \left(-\lambda\left(u_{1}-\theta_{\text {syn }}\right)\right)}\right)<0 .
$$

It means that in any cases, there is always the inequality

$$
g_{n}\left(u_{i}-u_{1}\right)\left(u_{1}-V_{\text {syn }}\right)\left(\frac{1}{1+\exp \left(-\lambda\left(u_{i}-\theta_{\text {syn }}\right)\right)}-\frac{1}{1+\exp \left(-\lambda\left(u_{1}-\theta_{\text {syn }}\right)\right)}\right)<0 .
$$

Therefore,

$$
\begin{aligned}
\frac{d \Phi(t)}{d t} & \leq \sum_{i=2}^{n} \int\left[a\left(u_{i}-u_{1}\right)^{2}\left(f^{\prime}\left(u_{1}\right)+\sum_{k=2}^{3} \frac{f^{(k)}\left(u_{1}\right)}{k !}\left(u_{i}-u_{1}\right)^{k-1}-\sum_{k=1, k \neq i}^{n} \frac{g_{n}}{1+\exp \left(-\lambda\left(u_{k}-\theta_{s y n}\right)\right)}\right)-b\left(v_{i}-v_{1}\right)^{2}\right] d x \\
& \leq \sum_{i=2}^{n} \int_{\Omega}\left[a\left(u_{i}-u_{1}\right)^{2}\left(M-\sum_{k=1, k \neq i}^{n} \frac{g_{n}}{1+\exp \left(-\lambda\left(u_{k}-\theta_{s y n}\right)\right)}\right)-b\left(v_{i}-v_{1}\right)^{2}\right] d x .
\end{aligned}
$$

If $N=\inf \left\{u_{i}(x, t), i=1,2, \ldots, n, x \in \Omega, t \geq 0\right\}, d_{u_{i}}=d_{u_{j}}, i, j=1,2, \ldots, n$ and $g_{n}>\frac{M\left[1+\exp \left(-\lambda\left(N-\theta_{\text {syn }}\right)\right)\right]}{n-1}$, then

$$
M-\sum_{k=1, k \neq i}^{n} \frac{g_{n}}{1+\exp \left(-\lambda\left(u_{k}-\theta_{s y n}\right)\right)} \leq M-\frac{(n-1) g_{n}}{1+\exp \left(-\lambda\left(N-\theta_{\text {syn }}\right)\right)}<0 .
$$

Finally, there is always another constant $\beta>0$, such that

$$
\frac{d \Phi(t)}{d t} \leq-\beta \Phi(t) \Rightarrow \Phi(t) \leq \Phi(0) e^{-\beta t}
$$


where,

$\beta=\min \left(\frac{2}{\varepsilon}\left[\frac{(n-1) g_{n}}{1+\exp \left(-\lambda\left(N-\theta_{\text {syn }}\right)\right)}-M\right], 2 b\right)$.

Thus, there is the synchronization if the coupling strength is verified such that $g_{n}>\frac{M\left[1+\exp \left(-\lambda\left(N-\theta_{\text {syn }}\right)\right)\right]}{n-1}$.

If $f$ is cubic, there is the following corollary.

Corollary 1. Suppose that $\mathrm{f}$ is a cubic function, $f(u)=m_{3} u^{3}+m_{2} u^{2}+m_{1} u+m_{0}$, where

$m_{3}, m_{2}, m_{1}, m_{0}$ are constants with $m_{3}<0$ and if

$g_{n}>\frac{1}{n-1}\left(m_{1}-\frac{m_{2}^{2}}{3 m_{3}}\right)\left(1+\exp \left(-\lambda\left(N-\theta_{\text {syn }}\right)\right)\right)$, the

network $S=\left(\left(u_{1}, v_{1}\right),\left(u_{2}, v_{2}\right), \ldots,\left(u_{n}, v_{n}\right)\right)$ synchronizes then in the sense of Definition 1.

\section{NUMERICAL SIMULATIONS}

In the following, the paper shows the numerical results obtained by integrating the system (4)

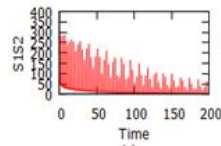

(a)

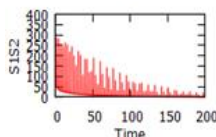

(f)
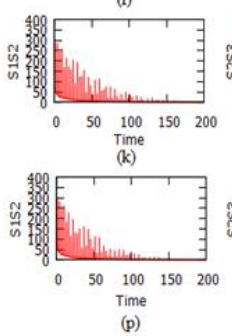

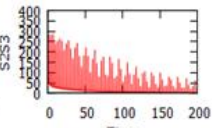

Time

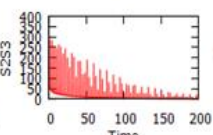

(g)

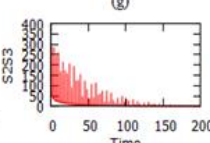

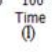

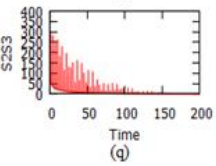

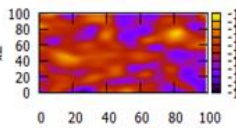

$\mathrm{x} 1$
(c)

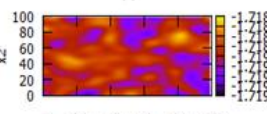

(h)

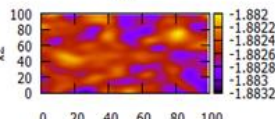

$\mathrm{x} 1$
$(\mathrm{~m})$

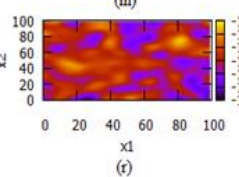

where $n=3, f(u)=-u^{3}+3 u$, and with the following parameter values: $a=1, b=0.001, c=0, \varepsilon=0.1, d_{u}=0.05$. The integration of system was realized by using $\mathrm{C}++$, on $[0 ; T] \times \Omega=[0 ; 200] \times[0 ; 100] \times[0 ; 100]$.

Figure 4 illustrates the phenomenon of synchronization. The simulations show that the system synchronizes from the value $g_{3}=0.025$. In the figures (a), (b), (f), (g), (k), (l), (p), (q), we represented the phase portraits $\left(u_{1}\left(x_{1}, x_{2}, t\right), u_{2}\left(x_{1}, x_{2}, t\right)\right)$ and $\left(u_{2}\left(x_{1}, x_{2}, t\right), u_{3}\left(x_{1}, x_{2}, t\right)\right)$ for $t \in[0 ; T]$ and for all $\left(x_{1}, x_{2}\right) \in \Omega$. It is observed (figure (p) and (q)) that for $g_{3}=0.025, u_{1}\left(x_{1}, x_{2}, t\right) \approx u_{2}\left(x_{1}, x_{2}, t\right)$ and $u_{2}\left(x_{1}, x_{2}, t\right) \approx u_{3}\left(x_{1}, x_{2}, t\right)$ for all $t \in[0 ; T]$ and for all $\left(x_{1}, x_{2}\right) \in \Omega$. In the figures (c), (d), (e), (h), (i), (j), (m), (n), (o), (r), (s), (t), they show the isovalues of $u_{i}\left(x_{1}, x_{2}, 190\right), i=1,2,3$. The results show that for $g_{3}=0.025$, the obtained patterns are "identical".
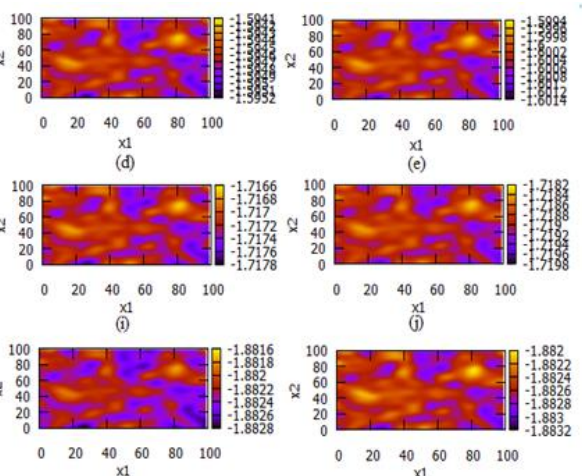

$x 1$
$(0)$

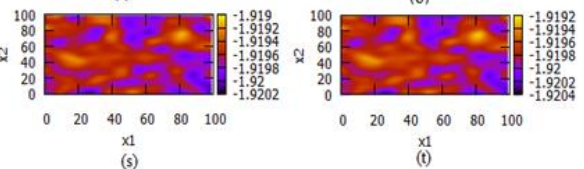

Figure 4. Synchronization of a complete network of three nonlinearly coupled "neurons"

The synchronization occurs for $g_{3}=0.025$. Before synchronization, for $g_{3}=0.01$ : the figure (a) represents the temporal dynamic of $u_{2}$ with respect to $u_{1}$, for all $\left(x_{1}, x_{2}\right) \in \Omega$; the figure (b) represents the temporal dynamic of $u_{3}$ with respect to $u_{2}$; the figure (c) represents the isovalues of $u_{1}\left(x_{1}, x_{2}, 190\right)$; similarly the figures (d) and (e) represent the isovalues of $u_{2}\left(x_{1}, x_{2}, 190\right)$ and $u_{3}\left(x_{1}, x_{2}, 190\right)$; the similar simulations are reproduced for $g_{3}=0.015$ (figures (f), (g), (h), (i), (j)), 
$g_{3}=0.023 \quad$ (figures $\left.(\mathrm{k}),(\mathrm{l}),(\mathrm{m}),(\mathrm{n}),(\mathrm{o})\right)$ and $g_{3}=0.025$ (figures (p), (q), (r), (s), (t)). For the value $g_{3}=0.025$, there is the synchronization of three "neurons"

The following research focuses on the minimal values of coupling strength $g_{n}$ to observe a phenomenon of synchronization between $n$ subsystems modeling the function of neuron network.
From the above result, in the case of three nonlinearly coupled neurons, for the coupling strength over or equal to $g_{3}=0.025$, these neurons have a synchronous behavior (Figure 4). By doing similarly for the complete networks of nonlinearly identical coupled neurons, the values of coupling strength according to the number of neurons $n$ are reported in Table 1.

Table 1. The minimal coupling strength necessary to observe the synchronization of $\mathbf{n}$ nonlinearly coupled neurons

\begin{tabular}{crrrrrrr}
\hline$n$ & & & $\mathbf{2}$ & $\mathbf{3}$ & $\mathbf{4}$ & $\mathbf{5}$ & $\mathbf{6}$ \\
\hline$g_{n}$ & & & 0.042 & 0.025 & 0.017 & 0.014 & 0.012 \\
$n$ & 7 & 8 & 9 & 10 & 11 & 12 & 13 \\
$g_{n}$ & 0.01 & 0.0094 & 0.009 & 0.0082 & 0.0078 & 0.0076 & 0.0072 \\
$n$ & 14 & 15 & 16 & 17 & 18 & 19 & 20 \\
$g_{n}$ & 0.0069 & 0.0068 & 0.0065 & 0.0063 & 0.0062 & 0.0061 & 0.006 \\
\hline
\end{tabular}

Following these numerical experiments, it is easy to see that the coupling strength required for observing the synchronization of $n$ neurons depends on the number of neurons. Indeed, the points in Figure 5 represent the coupling strength of synchronization according to the number of neurons in complete network, and the red curve represents the representative one

$$
g_{n}=\frac{0.038}{n-1}+0.004,
$$

where, $n$ is the number of neurons in network. Thus, the coupling strength necessary to obtain the synchronization of $n$ neurons follows this law.

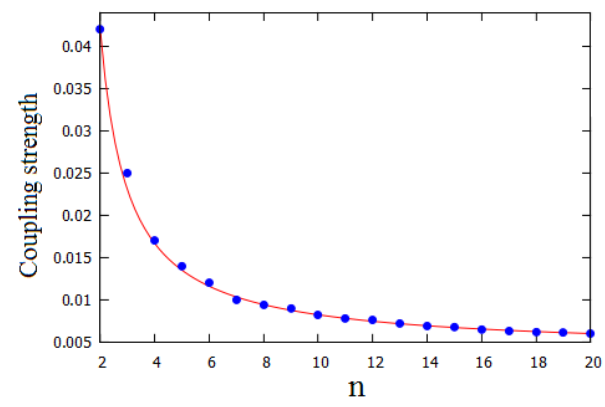

Figure 5. The evolution of the coupling strength $g_{n}$ for which the synchronization of $n$ neurons takes place according to the number $n$ nonlinearly coupled neurons in complete network and

$$
\text { it follows the law } g_{n}=\frac{0.038}{n-1}+0.004
$$

\section{CONCLUSIONS}

This study gave the sufficient condition on the coupling strength to achieve the synchronization in complete network of $n$ coupled systems of reaction-diffusion on Fitzhugh-Nagumo type. From Theorem 1, there is the result $g_{n}>\frac{M\left[1+\exp \left(-\lambda\left(N-\theta_{\text {syn }}\right)\right)\right]}{n-1}$ which shows that the bigger the value of $n$ is, the smaller the $g_{n}$ is. Numerically, it is seen that the synchronization is stable when the coupling strength exceeded to certain threshold and depends on the number of "neurons" in graphs. The bigger the number of "neurons" is, the easier the phenomenon of synchronization will be obtained. Then, a compromise between the theoretical and numerical results can be reached. In addition, it is necessary to conduct further studies on the different synchronization regimes in free network coupled by chemical synapse.

\section{REFERENCES}

Ambrosio, B., \& Aziz-Alaoui, M. A. (2012). Synchronization and control of coupled reaction-diffusion systems of the FitzHugh-Nagumo-type. Computers and Mathematics with Applications, 64, 934-943.

Ambrosio, B., \& Aziz-Alaoui, M. A. (2013). Synchronization and control of a network of coupled reactiondiffusion systems of generalized FitzHugh-Nagumo type. ESAIM, 39, 15-24. 
Aziz-Alaoui, M. A. (2006). Synchronization of Chaos. Encyclopedia of Mathematical Physics, Elsevier, 5, 213-226.

Belykh, I., De Lange, E., \& Hasler, M. (2005). Synchronization of bursting neurons: What matters in the network topology. Phys. Rev. Lett, 94, 188101.

Corson, N. (2009). Dynamique d'un modèle neuronal, synchronisation et complexité (doctoral dissertation). University of Le Havre, France.

Corson, N., \& Aziz-Alaoui, M. A. (2009). Complex emergent properties in synchronized neuronal oscillations. In From system complexity to emergent properties (pp. 243-259). Springer, Berlin, Heidelberg.

Ermentrout, G. B., \& Terman, D. H. (2009). Mathematical Foundations of Neurosciences. Springer.

Hodgkin, A. L., \& Huxley, A. F. (1952). A quantitative description of membrane current and its application to conduction and excitation in nerve. J. Physiol, 117, 500-544.
Izhikevich, E. M. (2007). Dynamical Systems in Neuroscience: The Geometry of Excitability and Bursting. Terrence J. Sejnowski and Tomaso A. Poggio The MIT Press, Cambridge, Massachusetts, London, England.

Keener, J. P., \& Sneyd, J. (2009). Mathematical Physiology: Systems Physiology, Second Edition. Antman S.S., Marsden J.E., and Sirovich L. Springer.

Murray, J. D. (2002). Mathematical Biology. I. An Introduction, Third Edition. Springer.

Nagumo, J., Arimoto, S., \& Yoshizawa, S. (1962). An active pulse transmission line simulating nerve axon. Proc. IRE, 50, 2061-2070.

Pikovsky, A., Rosenblum, M., \& Kurths, J. (2001). Synchronization, A Universal Concept in Nonlinear Science. Cambridge: Cambridge University Press, England. 